\title{
Is Hyaluronic Acid Gel a Good Option for Breast Augmentation?
}

\author{
Per Hedén
}

Received: 30 July 2010/ Accepted: 14 September 2010/Published online: 17 October 2010

(C) Springer Science+Business Media, LLC and International Society of Aesthetic Plastic Surgery 2010

We have clinical experience with Macrolane in body contouring dating back to 2002. Initially, it was used exclusively in clinical studies, but since the European conformity marking in 2007, it has found its place as a routine clinical treatment in our unit. It is agreed that the absorption time (albeit with great variability) is relatively quick in many patients, making the procedure expensive. The expense notwithstanding, this type of procedure is greatly in demand because many patients are uncertain about the outcome of a surgical procedure (e.g., a beast augmentation). It is not uncommon for patients undergo Macrolane augmentation and then after absorption of the material, convert to having breast implants. Thus, we see a new group of patients unwilling to undergo surgery for consultations.

Even if mammographic evaluation can be impaired after hyaluronic acid injections, radiologists (e.g.,. Dr. Magnus Tengvar) involved in our clinical studies have stated that ultrasound added to the mammography provides the same sensitivity and specificity as mammography alone for breasts without Macrolane. To facilitate radiologic examination, it is recommended that the material be placed more like an implant between the gland and muscle with our described pocket technique or in a partial submuscular position.
We also recommend that all Macrolane patients older than 40 years should have a mammogram taken less than 3 months before the treatment. Due to the current limited experience with larger series of breasts augmented with Macrolane, we also recommend avoiding treatment of patients with an increased risk for breast cancer.

In relation to the nodule and capsule formation treatment, this obviously is something the patient should be well informed about before deciding to undergo the treatment. However it also is important to underscore that treatment of these problems is simple and that in spite of these complications, very few patients $(<2 \%)$ request removal of the material.

In discussing the place for Macrolane in the clinical setting, it must be recognized that many other indications besides breast augmentation exist. In our experience, buttocks, calves, and male chest enhancements have provided patient satisfaction levels that equal those for breast augmentations.

Conflict of interest The author is a consultant and principal investigator in the preclinical studies of Macrolane for Q-Med Co.

P. Hedén $(\bowtie)$

Akademikliniken, Storängsv 10, 11542 Stockholm, Sweden

e-mail: Per.heden@ak.se 